\title{
Models on liver: alternative methods in hepatotoxicity
}

\author{
Francesca Caloni $^{1}$, Giovanna Mazzoleni ${ }^{2}$, Marisa Meloni ${ }^{3}$, Chiara Urani ${ }^{4}$, \\ Maura Ferrari ${ }^{5}$, Silvia Dotti ${ }^{5}$ and Yula Sambuy ${ }^{6}$ \\ ${ }^{1}$ Università degli Studi di Milano, Department of Health, Animal Science and Food Safety (VESPA), Milan, Italy; ${ }^{2}$ Università \\ degli Studi di Brescia, Department of Clinical and Experimental Sciences (DSCS), School of Medicine, Brescia, Italy; \\ ${ }^{3}$ VitroScreen, In vitro research Laboratories, Milan, Italy; ${ }^{4}$ University of Milan Bicocca, Department of Earth and Environmental \\ Sciences, Milan, Italy; ${ }^{5}$ Istituto Zooprofilattico Sperimentale della Lombardia e dell'Emilia Romagna "Bruno Ubertini" \\ (IZSLER), Brescia, Italy; ${ }^{6}$ CRA-NUT, Food \& Nutrition Research Center, Agricultural Research Council, Rome, Italy
}

http://dx.doi.org/10.14573/altex.1506181

On May 19, 2015, a meeting was held on the use of alternative methods for the evaluation of hepatotoxicity. It was chaired by Prof. Francesca Caloni and Prof. Giovanna Mazzoleni, and organized by CELLTOX, the Italian Association of in vitro Toxicology, in partnership with the Italian Reference Center for Alternative Methods, Welfare and Care of Laboratory Animal and MISTRAL (Integrated Models for Prevention and Protection in Environmental and Occupational Health) Research Center, University of Brescia, Italy. The meeting was hosted by the Istituto Zooprofilattico Sperimentale della Lombardia e Emilia Romagna, Brescia.

The conference was introduced by Dr Maura Ferrari, who reported on the activity of the Italian Reference Center for Alternative Methods, Welfare and Care of Laboratory Animals, indicating the need for alternative liver models.

Dr Emma Di Consiglio (ISS), in her presentation entitled "In vitro biokinetics: how to improve the predictive value of liver cell models," presented the overall strategy and the final conclusions reached by the European Project Predict-IV (EU-funded collaborative project \#202222), focusing on the relevance of biokinetics data for improving the prediction capability of in vitro tests and, in particular, of repeated dose studies performed on in vitro liver cell models. The overall aim of Predict-IV was to develop an improved testing strategy for drug safety during the discovery phase. Among the main target organs, the liver was shown to be mostly responsible for failure in drug development and drug withdrawal. The poor predictivity seen in certain animal experiments was also discussed. In this presentation, the use and applicability of the three liver cell models, i.e., primary rat hepatocytes, primary human hepatocytes and the human HepaRG cell line, considered and challenged with different model drugs, including ibuprofen and amiodarone, were described. The quantification of the parent compound and, where possible, its metabolites and their distribution in various in vitro compartments enabled the development of biokinetic models, from which it was possible to derive real in vitro exposure concentrations. More compounds need to be analyzed with a broader concentration range to fully exploit these promising results for improved prediction of hepatotoxicity and hazard assessment for humans.

Dr Barbara De Servi, VitroScreen, described the significant advances in the field of three-dimensional (3D) cellular models. In particular, cells cultured in 3D systems can contain more than one cell type, thus representing a more physiologically relevant microenvironment for tissue modelling, and develop cell junction patterns, which reflect in vivo structures better than 2D single cell models. The "microliver" represents a useful instrument in the development of pre-clinical studies for drug discovery, mechanistic toxicology, drug metabolism, cholestasis, toxicokinetics and intrinsic clearance prediction. Furthermore, the "microliver" can be a relevant model for investigating long-term and chronic toxicology of food ingredients, their mechanism of action and antioxidant activity, and for defining the hepatotoxicity of chemicals, pesticides and newly developed materials. Although the results obtained with the "microliver" model are promising and the model is gaining increasing relevance, some critical issues need to be underlined: in order to guarantee high data reproducibility, well-trained scientists have to be involved and particular attention has to be paid to the number of replicates depending on the specific endpoint.

Dr Annalisa Manenti described how biomedical technologies could be applied to toxicology and, in particular, to liver toxicology. The presentation started with a brief explanation of the bioengineering field and methodologies, and with a summary of the main skills and expertise of SKE Advanced Therapies ${ }^{\circledR}$, providing an insight into its main products. The presentation continued by focusing on 3D cell cultures: in particular, bioreactors were addressed extensively. Information 
was provided on how they are designed, their main elements and applications, and advantages offered by them in many fields of biomedical research ranging from tissue engineering to toxicology. Finally, it was shown how these technologies could be successfully applied to liver toxicology, and mention was also made of the possible advantages and challenges that still need to be addressed.

Prof. Giovanna Mazzoleni underlined the physiological importance of the liver and the impossibility of artificially substituting its multiple activities due to the species-specificity of its functions and to the inter-individual variability and susceptibility to injury, all of which collectively make animal models poorly informative/predictive for human pathophysiological and toxicological studies. In order to lessen and offset hepatotoxic effects, a large number of methods have been developed and are currently applied in risk assessment procedures and toxicological/epidemiolosgical studies aimed at identifying potential human hazards. Among them, in vitrolex vivo models of liver functions have shown significant growth in the last twenty years. However, at present, none of the commonly used models seem to be good enough to be considered a suitable model of the in vivo organ. New approaches are thus required in order to develop physiologically significant models that, recapitulating critical liver functions, can be relevant to humans. The more promising results seem, at present, to be derived from 3D culture techniques, which, by guaranteeing the preservation of at least some characteristics of the complex hepatic microenvironment, can favor cell survival and in vitro expression of the liver-specific differentiated phenotype. As a basis for the development of innovative liver models, tissue engineering approaches and methods, coupled with the possibility/potential of new emerging micro-/nanotechnologies, and the relevance of 3D bio-constructs as reliable liver analogues were discussed. Particular attention was paid to model systems based on the use of fluid-dynamic microenvironments (bioreactor systems) and, more specifically, to the use of the microgravity-based Rotary Cell Culture System (RCCS ${ }^{\mathrm{TM}}$, Synthecon, Inc.) device.

Dr Alfonso Lostia's, (EURL-ECVAM) presentation described the scientific strategy and reasoning process, derived from the knowledge of relevant Adverse Outcome Pathways (AOPs), that was used to design a case study aimed at predicting repeated dose liver toxicity in the SEURAT-1 EU project. The objective of this case study was to develop and compare the performances of several AOP-based classification models aimed at distinguishing between hepatotoxicants and nonhepatotoxicants, with hepatotoxicity being mainly related to three major liver adverse outcomes associated with repeated dose exposure: cholestasis, fibrosis and steatosis. Within SEURAT-1, HepaRG, a well-characterized in vitro liver system, was exposed to 90 selected reference compounds $(75 \%$ known hepatotoxicants and $25 \%$ known non-hepatotoxicants based on in vivo evidence), and the knowledge of the key events of the three AOPs was used to select the in vitro endpoints to be measured. High-throughput screening (using a 96-well plate format) was employed to test the reference chemicals, and the read-outs of the selected in vitro endpoints were performed using high content screening based mainly on automated imaging. The selection of reference chemicals depended on whether or not they caused hepatotoxicity based on animal and/or human data. To this end, along with cosmetic chemicals, pharmaceuticals, pesticides and environmental chemicals were also included in the dataset. The goal was to minimize false negative predictions while ensuring adequate discrimination between hepatotoxic and non-hepatotoxic chemicals. The resulting classification model(s) were foreseen to be useful for the hazard profiling of large chemical sets and priority setting (to be tested further). 